\title{
Utiliser Linkedln en entreprise et hors-entreprise : entre stratégie collective et individuelle
}

\section{To use LinkedIn within the organisation and outside: between individual and collective strategies}

\author{
Hélène Hoblingre Klein ${ }^{1}$ \\ ${ }^{1}$ Laboratoire interuniversitaire des sciences de l'éducation et de la communication, Université de Strasbourg, \\ helene.hoblingre@insa-strasbourg.fr
}

\begin{abstract}
RÉSUMÉ. Les professionnels des ressources humaines utilisent Linkedln en entreprise (en interne) à la fois pour le recrutement et pour le "développement de la marque employeur ». Hors-entreprise (en externe) tout le monde peut s'adonner sur le réseau à des activités de recherche d'emploi et de " développement de sa marque personnelle ». Un questionnaire et dix entretiens montrent que les $\mathrm{RH}$ poursuivent donc à la fois une stratégie individuelle tournée vers l'affirmation de leur "identité professionnelle personnelle» et une stratégie collective au service de l'efficacité. La stratégie collective peut alors inhiber l'expression de référents identitaires au détriment de la stratégie individuelle. La faculté de pouvoir concilier ces deux pôles stratégiques dépend en grande partie du type d'activité pratiqué : la conciliation est davantage possible lorsque l'activité relève de l'action que lorsqu'elle relève de l'affichage d'informations sur le profil. S'afficher ouvertement au nom d'un collectif tout en réalisant secrètement, " en sous-marin », un travail de réseautage et de communication interpersonnelle pour son propre compte s'impose également comme une technique de conciliation.

ABSTRACT. People who work for human resources (HR) use Linkedln within the organisation (internally) both for recruiting and for employer branding. Outside the organisation (externally) everybody can use Linkedln to find a job or for personal branding. One survey and ten interviews show that HR pursue two strategies. The first one is individual: the purpose is to assert one's "professional personal identity". The second one is collective: the purpose is to be efficient. Thus, the collective strategy can inhibit the expression of elements that refer to identity, thus ruining individual strategy to the detriment of individual strategy. The possibility to match these two strategic aspects mainly depends on the activity which is practised. Conciliating these two aspects is more possible when the activity relies on action rather than on showing information on the profile. Another technique for conciliating these two aspects is possible: the user can openly give information in the name of the company; and at the same time, "as a submarine" he can develop his social network secretly and also communicate with other people.
\end{abstract}

MOTS-CLÉS. Réseau social numérique professionnel, stratégie individuelle, stratégie collective, employer branding, personal branding, identité professionnelle, référents identitaires, profil.

KEYWORDS. Professionnal social network, individual stratégie, collective strategie, employer branding, personal branding, professionnal identity, identity referents, profil.

\section{Introduction}

L'utilisation des réseaux sociaux en interne pour le travail s'est accrue de manière significative ces dernières années. Une enquête menée auprès de 3249 cadres au niveau international a révélé que deux tiers d'entre eux utilisent le Web 2.0 pour travailler et qu'un nombre croissant d'entreprises s'investissent dans les réseaux sociaux et les blogs (Bughin \& Chui, 2010). Deux nouvelles dénominations permettant de désigner les entreprises ayant recours de manière intensive au Web 2.0 sont ainsi apparus : les networked entreprises et les «entreprises 2.0 ». Les networked entreprises tentent, à l'aide du Web 2.0, de relier les efforts internes des salariés pour trouver de nouveaux clients, de nouveaux partenaires voire de nouveaux fournisseurs. Quant aux entreprises 2.0, Andrew McAfee (co-directeur du centre MIT pour les entreprises numériques) les décrit ainsi : ce sont des entreprises faisant « une utilisation de plateformes sociales émergentes au sein de sociétés ou entre des sociétés, leurs partenaires et leurs clients » (MacAfee, 2006). 
Dans ce contexte, nombre d'entreprises ont recours au réseau social numérique professionnel (RSNP) LinkedIn pour des activités internes : il s'agit par exemple de « communiquer autour de leur marque, nouer des partenariats, élargir un circuit de distribution, améliorer la gestion de la relation client, favoriser la collaboration interne [...] recruter des collaborateurs » (Girard, 2012, p. 14). Ici, l'expression activité interne me permettra de désigner ces activités réalisées en entreprise, c'est-à-dire dans un contexte interne. Il existe également sur LinkedIn tout un pan d'activités externes, autrement dit des activités réalisées en dehors du cadre d'une structure, dans un contexte externe. Les activités de recherche d'emploi ou auto-entrepreneuriales rentrent dans cette catégorie. Les termes « internes » et « externes » me permettront donc de mettre la focale sur les auteurs/acteurs de l'activité et non pas sur la population visée par cette activité. Cette perspective diffère de celle des chercheurs en management qui s'intéressent à l'externalité ou à l'internalité du public (Girard, Fallery, \& Rodhain, 2011).

Les recherches en économie et gestion (Girard, 2012), en communication professionnelle (Roberts $\&$ Roach, 2008, p.111) et en information-communication (Larroche, 2014) font apparaitre la dimension stratégique des activités internes, orientées tendanciellement vers un objectif collectif d'efficacité. Par ailleurs, l'analyse des phénomènes de mise en scène sur les réseaux sociaux (Coutant \& Stenger, 2010), (Cardon, 2008) ainsi que les recherches sur l'identité professionnelle (Beckers, 2007) et sur les stratégies identitaires (Taboada-Leonetti, 1998) permettent d'adopter le point de vue selon lequel les activités externes servent des objectifs stratégiques tendanciellement individuels (évolution de carrière, employabilité...) orientés vers l'affirmation de ce que nous décrirons plus loin comme étant l'« identité professionnelle personnelle ».

Cependant, les sciences de l'éducation et les sciences de l'information et de la communication n'ont pas approfondi l'analyse des réseaux sociaux professionnels sous l'angle de la conciliation entre activité externe et activité interne. Les articles de Cindy Felio sur la régulation de l'usage des TIC au travail (Boudokhane-Lima \& Felio, 2015), (Felio, 2016) mettent l'accent sur les problèmes qu'une telle utilisation peut impliquer par rapport à l'intrusion du professionnel dans le privé, en terme d'organisation du temps et de l'espace, mais la réflexion sur la manière dont une activité interne s'articule ou s'oppose avec une activité externe reste en suspens. La possibilité pour les utilisateurs de LinkedIn de s'engager simultanément dans deux types de stratégie (les unes en lien avec des activités internes, les autres en lien avec des activités externes) s'impose donc comme une zone de recherche inexplorée.

$\mathrm{Au}$ travers d'une approche théorique et d'une enquête qualitative, j'initierai dans cet article une analyse compréhensive permettant d'envisager de quelles manières les stratégies internes et externes s'excluent ou se combinent lorsque l'individu utilise LinkedIn comme outil de travail au sein d'une collectivité. Plus exactement, je tenterai de répondre à la question suivante : peut-on déployer sur un seul et même réseau une stratégie au service de l'efficacité collective (perspective interne) et une stratégie au service de la mise en scène de son identité professionnelle personnelle (perspective externe), ou existe-t-il un clivage entre ces deux catégories ? Cette contribution s'inscrit donc dans le prolongement des travaux de Stenger et Coutant mettant en avant la tension sur les RSN entre " pression de conformité sociale et volonté de se présenter comme original en refusant de s'y rendre » (Coutant \& Stenger, 2010, p. 5). Elle concerne donc la manière dont l'ancrage dans une activité collective de travail sur les RSNP affecte la négociation de la « face », laquelle est définie par Goffman comme «la valeur sociale positive qu'une personne revendique effectivement à travers une ligne d'action que les autres supposent qu'elle a adoptée au cours d'un contact particulier » (Goffman, 1974, p. 9). 


\section{Cadre théorique}

\subsection{Choix définitionnels}

La « stratégie » est couramment définie comme un « ensemble d'actions coordonnées, d'opérations habiles, de manœuvres en vue d'atteindre un but précis ${ }^{1}$. Dans une perspective comportementaliste, le terme stratégie désignera ici un ensemble de comportements, d'usages et de pratiques tournés vers un but particulier. Ce terme est d'autant plus adapté qu'il est utilisé abondamment dans les recherches portant sur la visibilité numérique. Les auteurs évoquent la visibilité et l'invisibilité comme stratégies (Felio, 2013) (Stenger \& Coutant, 2010) et les débats sur la Privacy révèlent une gestion stratégique de l'identité numérique qui consiste à afficher certains aspects de son identité et à en masquer d'autres (Cardon, 2013).

Sur cette base, l'expression stratégie collective me permettra de désigner les stratégies qui se déploient dans un contexte organisationnel et plus particulièrement dans le cadre d'un emploi. C'est le cas par exemple lorsque le réseau est utilisé comme outil de recrutement ou de prospection commerciale. Plus égocentrées, les stratégies individuelles renverront aux stratégies servant la réalisation des projets du stratège. Les stratégies individuelles peuvent donc concerner tous les utilisateurs de LinkedIn sans distinction: salariés, étudiants, individus sans emploi, autoentrepreneurs... Elles relèvent par exemple de la quête d'un emploi, d'une volonté de changement professionnel ou de valorisation de soi, manifestations du seul désir et du seul besoin de l'individu.

\subsection{Gagner en efficacité et asseoir son identité : deux objectifs stratégiques sur Linkedln}

\subsubsection{Gagner en efficacité, un objectif tendanciellement collectif}

En interne, les réseaux sociaux numériques (RSN) notamment professionnels sont devenus des outils incontournables pour répondre aux exigences de performance des grandes entreprises concernant le recrutement, le commerce, le marketing et la gestion réputationnelle. Novametrie, un institut spécialisé dans les études stratégiques européenne, a démontré que « $80 \%$ des directeurs des ressources humaines et $92 \%$ des collaborateurs associaient les réseaux sociaux à des outils d'image et de communication $»^{2}$ en 2009. Plus tard, en 2012, Opinion Way comptabilise la présence de $95 \%$ des entreprises françaises sur les réseaux sociaux (Kriegk, 2012). Face à cette montée en puissance des réseaux sociaux comme outils stratégiques, la question de l'évaluation de leur performance a émergé. D'après Dominique Rouziès (Professeur titulaire de la Chaire EDF à HEC Paris) « la question de la mesure de la performance des medias sociaux dans les activités commerciales est une question centrale pour de nombreux acteurs $\gg{ }^{3}$.

Or, LinkedIn constitue un outil particulièrement adapté pour les activités de recrutement, commerce, marketing et gestion réputationnelle. Des entreprises utilisent ce réseau comme outil de travail (Skeels \& Grudin, 2009), par exemple pour coordonner leurs activités (Weinstein, 2010), pour le recrutement ou le commerce (Thew, 2008). Outillé pour les activités de sourcing et de prospection, LinkedIn est décrit par plusieurs auteurs comme un réseau fortement axé sur le développement de relations commerciales (Malita, Badescu, \& Dabu, 2010, p. 3072), (Roberts \& Roach, 2008, p.111) 4 . Il permet par exemple de se livrer à des activités de prospection tout en préservant son anonymat afin d'éviter d'être vu, notamment par ses concurrents en cas de veille concurrentielle (Crussière, 2015). Les

\footnotetext{
${ }^{1}$ Source : Trésor de la langue française informatisé

${ }^{2}$ http://www.hec.fr/Grande-Ecole-MS-MSc/Actualites/2015-03-27-Les-medias-sociaux-un-puissant-outil-de-performancecommerciale

${ }^{3}$ http://www.ladn.eu/nouveaux-usages/etude-marketing/medias-sociaux-organisations-commerciales-croissance-et-performance/

${ }^{4}$ " social networking Web sites (SNWs) that focus more on business connections, such as Linkedln » (Roberts \& Roach, 2008, p.111) 
concepteurs de Linkedln ont d'ailleurs cherché à développer l'attractivité du site pour les commerciaux en fondant un service publicitaire en interne : Linkedln Ads (Chartier, 2013, p. 217).

Plus spécifiquement, LinkedIn est devenu un canal de recrutement privilégié. Entre 2013 et 2014, ce RSNP était déjà perçu comme un réseau d'avenir pour les recruteurs. D'abord en 2011, des chercheurs citent le cas d'une entreprise comptant s'investir rapidement dans LinkedIn pour le recrutement (Girard, Fallery, \& Rodhain, 2011). Plus tard, en 2014, LinkedIn est décrit comme un outil de recrutement pour «les recruteurs innovant » (Larroche, 2014). Certes, il peut y avoir un écart entre la perception de LinkedIn par les recruteurs et son utilisation effective : 77\% d'entre eux le perçoivent comme un «moyen de recrutement innovant et performant» mais seulement 67\% l'utilisent (Agostinelli, Arvanitakis, \& Ouvrard, 2015, p. 11). Malgré cet écart entre les velléités d'utilisation de LinkedIn et le recours réel à ce réseau pour le recrutement, le succès que rencontre LinkedIn pour ces activités est évident. Le site est décrit comme un réseau particulièrement utilisé comme outil de vérification de référence dans les ressources humaines (Roberts \& Roach, 2008, p.111) et au premier trimestre de l'année 2016, les produits, les formations et les services pour le recrutement représentaient la principale source de revenus pour LinkedIn, à hauteur de 557,647 millions de dollars (source : LinkedIn) ${ }^{5}$. Enfin, LinkedIn offre un service de publication d'offres d'emploi spécialement dédié aux recruteurs, et qui présente pour spécificité d'être en quelque sorte hybridé avec les fonctionnalités du réseau proprement dit puisque «la liste des personnes qui travaillent dans la société et qui sont membres du réseau du candidat est ajoutée [à l'offre d'emploi]» (Larroche, 2015).

A partir de ces différentes constatations on peut poser le postulat de base selon lequel LinkedIn est utilisé collectivement dans une perspective stratégique d'efficacité au travail, le terme "efficacité » désignant ici une manière de produire un effet attendu en termes de performance au travail, dans un contexte interne.

\subsubsection{Asseoir son identité professionnelle personnelle : un objectif stratégique individuel}

L'utilisation de LinkedIn en externe semble davantage répondre à un souci de mise en scène de son identité plutôt que d'efficacité. En effet, les recherches de Dominique Cardon, Alexandre Coutant et Thomas Stenger ont mis en évidence des liens existant entre mise en scène de soi sur les réseaux sociaux et identité : Dominique Cardon aborde la mise en scène de l'identité numérique comme un « design de la visibilité » (Cardon, 2008); Coutant et Stenger appréhendent l'exposition de soi sur les RSN comme un prélude à l'exposition identitaire (Coutant \& Stenger, 2010).

Au niveau conceptuel, l'identité possède un double statut : «il s'agit d'un fait de conscience, subjectif, donc individuel [...] mais il se situe aussi dans le rapport à l'autre, dans l'interactif » (Taboada-Leonetti, 1998, p. 43). L'identité n'est donc pas l'émanation du seul individu qui en est détenteur: elle peut également procéder de l'identité, des attentes et des représentations d'autres individus. Ce fait est aisément observable sur les RSN : les membres du réseau d'un utilisateur apportent une reconnaissance qui crédibilise certains éléments de l'identité mise en scène. Par exemple, on remarque que l'information publiée sur un profil est en partie contrôlée par les membres du réseau (Kluemper \& Rosen, 2009, p.570).

Le double statut du concept d'identité a ainsi amené Erving Goffman à dissocier l'identité sociale virtuelle, correspondant à l'identité pour autrui, et l'identité sociale réelle, correspondant à l'identité pour soi (Goffman, 1975). Il souligne que ces deux facettes ne sont pas toujours en correspondance. De son côté, Taboada-Leonetti distingue deux facettes: l'identité personnelle et l'identité collective. L'identité personnelle correspond à « ce par quoi l'individu se perçoit unique et différent de tous les autres » (Taboada-Leonetti, 1998, p. 45) tandis que l'identité collective repose sur les statuts que l'individu « partage avec d'autres membres d'un groupe social » (ibid. ).

\footnotetext{
${ }^{5}$ http://www.silicon.fr/linkedin-reprend-des-couleurs-au-1er-trimestre-2016-146218.html
} 
Je définirai donc «l'identité professionnelle personnelle» comme ce par quoi le professionnel se perçoit unique et différent du collectif de travail auquel il appartient. Quant à l'identité professionnelle collective, je la considère comme étant façonnée par les représentations du collectif professionnel auquel l'individu appartient. Ce faisant, je me positionne dans l'héritage d'une traditions théorique qui disloque le concept d'identité entre « un pôle individuel » et « un pôle structurel ou collectif » (Dubar, 1992, p. 509).

La manifestation de l'identité professionnelle personnelle sur LinkedIn peut être analysée grâce à l'identification des référents qui la constituent. En effet, l'identité professionnelle s'appuie sur «les référents du Curriculum Vitae et des activités professionnelles » (Mucchielli, 2013, p.14). Or, nombre de ces référents, listés et catégorisés par Alex Muccchieli (Mucchielli, 2013, p.13-14), apparaissent sur le profil LinkedIn (tableau 1). L'individu peut par exemple revendiquer et/ou manifester sur son profil des compétences et des qualités, notamment grâce à la partie «top compétences » ou grâce à l'exposition de réalisations concrètes. Or, on sait que l'affirmation du sentiment de compétence est une visée du « processus de constitution et de transformation de l'identité professionnelle » (Beckers, 2007, p. 169). De plus, en se proclamant publiquement membre d'une profession et en incluant ses pairs dans son réseau, l'utilisateur indique son appartenance à un milieu professionnel crédibilisant les informations données. Et pour cause, les recruteurs peuvent « trouver des indices de l'obtention d'un diplôme en analysant le réseau du candidat » (Larroche, 2015, p.150).

\begin{tabular}{|c|c|c|c|}
\hline $\begin{array}{c}\text { Catégorie de } \\
\text { référents }\end{array}$ & Sous-catégorie de référents & Référents & $\begin{array}{c}\text { Section du profil faisant } \\
\text { apparaître ces référents }\end{array}$ \\
\cline { 5 - 5 } Culturels & Système culturel & Système de valeurs & Centre d'intérêt / Résumé \\
\hline Culturels & Système culturel & Niveau d'éducation & Formation \\
\hline Psychosociaux & Références sociales & $\begin{array}{c}\text { Nom / Sexe / } \\
\text { Profession }\end{array}$ & En-tête du profil \\
\hline Psychosociaux & Références sociales & $\begin{array}{c}\text { Activité } \\
\text { Rôles sociaux }\end{array}$ & $\begin{array}{c}\text { Expériences / Expériences } \\
\text { de bénévolat / Projets }\end{array}$ \\
\hline Psychosociaux & Attributs de valeur sociale & $\begin{array}{c}\text { Qualités / Résultats / } \\
\text { Estimations diverses }\end{array}$ & $\begin{array}{c}\text { Résumé / Réalisations / } \\
\text { Prix et distinctions }\end{array}$ \\
\hline Psychosociaux & Potentialités de devenir & Motivation & Résumé \\
\hline Psychosociaux & images identitaires venant & Opinions d'autres \\
d'autres acteurs & individus & $\begin{array}{c}\text { Compétences / } \\
\text { Recommandations }\end{array}$ \\
\hline Psychosociaux & affiliations & $\begin{array}{c}\text { Groupes } \\
\text { d'appartenance }\end{array}$ & $\begin{array}{c}\text { Groupes / Abonnements / } \\
\text { Organisations }\end{array}$ \\
\hline
\end{tabular}

Tableau 1. Référents identitaires présents sur le profil Linkedln selon la catégorisation d'Alex Mucchielli

\subsubsection{Deux catégories d'activités au service de l'identité professionnelle personnelle sur Linkedln}

Le concept récent de personal branding (développement de sa marque personnelle) renvoie précisément à une gestion de de l'identité professionnelle personnelle. En effet, les chercheurs en

\footnotetext{
${ }^{6}$ En début d'année 2017 la plateforme Linkedın a été l'objet de modifications majeures. Les recherches présentés dans cet article ont été menée avant ces modifications. Pour cette raison j'ai repris dans ce tableau les intitulés des différentes sections du profil tels qu'ils étaient avant 2017.
} 
management des ressources humaines distinguent deux étapes nécessaires au personal branding: « définir l'identité de sa marque personnelle - c'est-à-dire un positionnement souhaité qui s'appuie sur ses compétences distinctives - et [...] communiquer ce positionnement, via un ou plusieurs profils » (Benraïss-Noailles \& Viot, 2012, p.137). C'est donc bien d'une gestion de l'identité professionnelle à un niveau personnel dont il s'agit ici. De ce fait, le «personal branding » implique une démarche individuelle : « travailler sa notoriété personnelle pour acquérir une plus grande crédibilité » (Delcroix, 2010, p. 101) ou veiller à «la mise en scène de soi » (Gozlan, 2014, p. 55) en ayant recours aux réseaux sociaux, aux blogs, aux sites personnels ou encore aux $\mathrm{CV}$ thèques.

A partir des deux étapes de personal branding mises en évidence par Benraïss-Noailles et Viot j'ai distingué deux catégories d'activité pouvant permettre d'asseoir son identité professionnelle personnelle en externe sur LinkedIn :

- La première (activité 1) relève de l'action : il s'agit de communiquer et d'élargir son réseau

- La deuxième (activité 2) relève du déclaratif, de l'affichage : elle concerne la publication d'un texte valorisant pour l'individu sur le profil LinkedIn

\subsection{Activités réalisées en interne dans une perspective d'efficacité chez les RH}

Comme je l'ai démontré plus haut, les activités réalisées en interne dans une perspective stratégique d'efficacité concernent largement les professionnels RH, ce qui m'a poussé à m'intéresser ici aux actions de ce public spécifique.

\subsubsection{Recrutement}

De manière générale, LinkedIn est utilisé pour les activités de sourcing, c'est-à-dire pour l'approvisionnement en produits, en services (Roll, 2010) ou en personnes. Plus spécifiquement, dans le domaine des ressources humaines, le sourcing « décrit tous les processus permettant de repérer et d'attirer des candidats » (Fondeur, 2006, p.48). Dans ce cadre, LinkedIn permet de mettre en œuvre un recrutement que l'on peut qualifier de stratégique, car le réseau est utilisé par un certain type d'entreprise pour rechercher une catégorie spécifique de candidats : le recrutement est majoritairement focalisé sur " des postes de cadres supérieurs orientés à l'international » (Girard, 2012, p.173) et la taille aussi bien que le secteur de l'entreprise déterminent l'utilisation qui est faite ou non de LinkedIn pour recruter.

\subsubsection{Marketing du recrutement et employer branding}

Véritable ramification des activités de recrutement, le marketing du recrutement se distingue cependant nettement du sourcing, car il consiste en un travail de gestion de l'image véhiculée par le groupe, dans l'optique d'attirer de nouveaux talents. Il constitue ce faisant une manière plus innovante de recruter, puisqu'il vise à attirer les candidats et non pas à aller les chercher. En cela, le marketing du recrutement peut être perçu comme une variante RH de l'approche «d'inbound marketing », laquelle consiste à faire venir le client à soi. Certains blogueurs affirment d'ailleurs que « recrutement inbound et marketing ne feront [bientôt] plus qu'un» (Audrerie, 2015) ou que l'apparition de "l'inbound recruiting » 7 (2016, site DW inbound) s'est faite conjointement à celle du recruteur 2.0.

Avec le marketing du recrutement, le recrutement n'est donc plus seulement appréhendée comme une recherche de candidats mais comme un processus de mise en scène qui, ayant des effets en termes d'image, permet d'attirer ces derniers. Et pour cause, les chercheurs en management et en psychologie des organisations ont déjà démontré que la qualité du processus de recrutement impacte l'image qu'aura le candidat de l'entreprise et l'attractivité de celle-ci (Lievens, van Dam, \& Anderson, 2002, p. 595). C'est notamment la rencontre entre les valeurs, les attentes de l'individu et celles de l'entreprise

\footnotetext{
${ }^{7}$ https://blog.digitaweb.com/inbound-recruiting/recruter-avec-inbound-recruiting
} 
qui vont impacter positivement l'image renvoyée par celle-ci (Backhaus, 2004). Dès lors, les contacts qu'auront eux les candidats avec l'entreprise et leurs ressentis par rapport au processus de recrutement constituent la «vitrine de la marque employeur» (RégionsJob, 2013, p. 5). Les organisations qui souhaitent attirer des talents ont donc tout intérêt à développer ce qui peut permettre au candidat de s'identifier à elle et à veiller à la qualité des contacts recruteur-candidat. Une enquête prenant pour terrain d'étude le site de recrutement Monster (Backhaus, 2004) a ainsi révélé que l'intégration de descriptions élogieuses aux offres d'emploi constituait une technique de développement de la marque employeur (employer branding) dans une perspective de marketing du recrutement.

\subsubsection{Deux catégories d'activités au service de l'efficacité sur LinkedIn}

Sur ces bases théoriques j'ai distingué deux catégories d'activité pouvant servir des objectifs d'efficacité en interne :

-La première (activité 3) relève de l'action : il s'agit d'utiliser LinkedIn pour recruter, prospecter des clients. Elle correspond à ce que j'appelle plus loin « l'utilisation comme outil de travail ».

- La deuxième (activité 4) relève du déclaratif, de l'affichage : elle concerne la publication d'un texte valorisant pour l'employeur sur le profil LinkedIn, dans une perspective de marketing employer et plus largement d'employer branding. Ce texte peut alors permettre d'attirer de nouveaux clients ou des candidats. En effet, les profils LinkedIn comportent une section "résumé » où il est possible d'afficher les différents avantages inhérents aux entreprises. Sur le profil d'un directeur des ressources humaines (DRH) on peut ainsi lire :

«Qu'offrons-nous ? D'excellents programmes de formation! un programme de mobilité à l'international ; des possibilités de télétravail ; d'agréables moments de détente en équipe; une super terrasse pour les pauses café ; tous les jours, c'est Friday wear ! Visitez notre page Facebook pour voir toutes les offres d'emploi disponibles. 8»

\subsection{Synthèse}

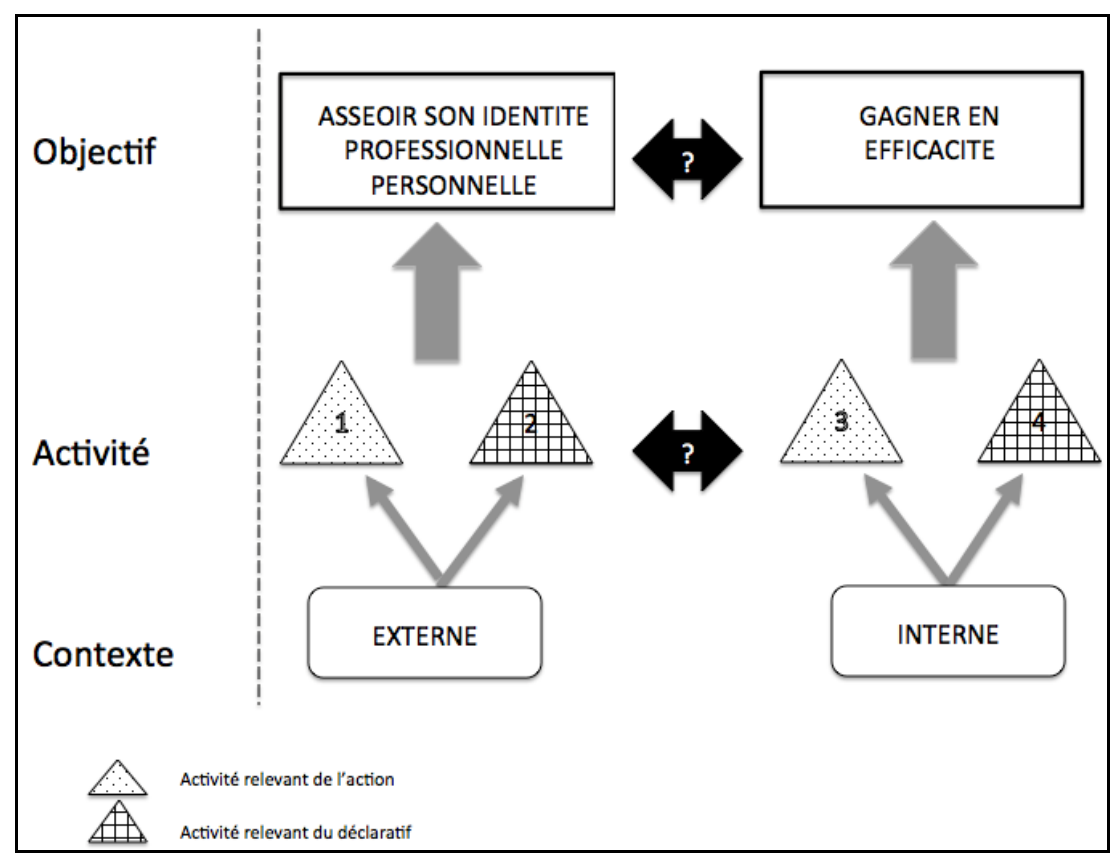

Figure 1. Problématique générale

La problématique s'articulera selon deux niveaux principaux : l'un relève des objectif et l'autre des activités stratégiques (figure 1). L'enjeu de cette analyse est donc d'une part de comprendre si une

\footnotetext{
${ }^{8}$ Traduction personnel d'un texte rédigé en anglais et publié sur le profil Linkedın d'un DRH en juin 2017
} 
stratégie au service de l'efficacité collective (perspective interne) est conciliable avec une stratégie au service de la mise en scène de son identité professionnelle personnelle (perspective externe), et d'autre part d'appréhender les combinaisons ou les incompatibilités entre deux catégories d'activités (1et $2 ; 3$ et 4) rattachées chacune à un contexte et un objectif déterminé.

\section{Méthodologie}

\subsection{Questionnaire à large échelle}

Un questionnaire préalable de recherche (tableau 2) a été élaboré selon les trois objectifs suivants : premièrement identifier une population impliquée en interne dans les activités 3 et 4 ; deuxièmement identifier pour chaque activité 3 et 4 une tendance stratégique (soit gagner en efficacité au travail soit asseoir son identité professionnelle personnelle); troisièmement appréhender les tendances relatives à la conciliation ou à l'opposition de deux pôles stratégiques rattachés chacun à un contexte particulier : gagner en efficacité (contexte interne) et asseoir son identité professionnelle (contexte externe).

\begin{tabular}{|c|c|c|}
\hline $\begin{array}{l}\text { Objectif des } \\
\text { questions }\end{array}$ & $\begin{array}{l}\text { Activité } \\
\text { concernée }\end{array}$ & Questions \\
\hline \multirow{2}{*}{$\begin{array}{l}\text { Identification de } \\
\text { deux activités } \\
\text { pratiquées en } \\
\text { internes }\end{array}$} & 3 & $\begin{array}{l}\text { 1. Dans le cadre de votre emploi actuel, utilisez-vous LinkedIn comme } \\
\text { outil de travail (ex : pour recruter, pour prospecter des clients ...)? }\end{array}$ \\
\hline & 4 & $\begin{array}{l}\text { 2. Dans le cadre de votre emploi actuel, avez-vous ajouté une description } \\
\text { de l'entreprise à laquelle vous appartenez sur votre profil LinkedIn? }\end{array}$ \\
\hline \multirow{2}{*}{$\begin{array}{l}\text { Estimation du } \\
\text { caractère } \\
\text { stratégique de } \\
\text { l'activité }\end{array}$} & 3 & $\begin{array}{l}\text { 3. Au travers de l'utilisation de LinkedIn comme outil de travail, espérez- } \\
\qquad \begin{array}{c}\text { vous : } \\
\text { - gagner en efficacité au travail ? } \\
\text { - asseoir votre identité professionnelle? }\end{array}\end{array}$ \\
\hline & 4 & $\begin{array}{l}\text { 4. L'ajout d'une description de l'entreprise à laquelle vous appartenez sur } \\
\qquad \begin{array}{r}\text { votre profil LinkedIn vous permet-il : } \\
\text { - de gagner en efficacité au travail ? } \\
\text { - d'asseoir votre identité professionnelle }\end{array}\end{array}$ \\
\hline
\end{tabular}

Tableau 2. Questionnaire préalable

\subsection{Entretiens directifs et semi-directifs}

\subsubsection{Entretiens principaux}

L'objectif des entretiens principaux était de comprendre si le pôle stratégique « asseoir son identité professionnelle» est plus facilement conciliable avec l'activité 3 ou avec l'activité 4. La question suivante a donc été soumise aux interviewés : "existe-t-il des tensions entre l'utilisation de LinkedIn par rapport à votre employeur (pour valoriser l'entreprise ou pour être plus efficace au travail) et l'utilisation de LinkedIn pour vous-même ? ». L'analyse des résultats a ensuite été notamment axée sur l'identification de marqueurs identitaires.6

\subsubsection{Entretiens complémentaires}

2 entretiens complémentaires venus clore cette enquête principale. Ils ont été réalisés auprès d'« experts LinkedIn », c'est-à-dire des travailleurs indépendants formant des clients à l'utilisation de ce réseau et revendiquant une expertise se manifestant au travers de la publication d'articles grand 
public ou de guides pratiques relatifs à LinkedIn. L'objectif était ici de confirmer les résultats de l'enquête principale et d'ouvrir de nouvelles perspectives en termes de recherche ou de réponses.

La question formulée était la suivante : "Chez vos clients, avez-vous constaté qu'il peut exister des tensions entre l'utilisation de LinkedIn par rapport à l'entreprise où on travaille (pour valoriser son employeur ou pour être plus efficace au travail) et l'utilisation de LinkedIn pour soi-même ? Comment dépasser cet éventuel clivage?»

\section{Résultats des enquêtes}

\subsection{Résultats du questionnaire préalable}

\subsubsection{Population concernée par les activités 3 et 4 : les professionnels $R H$}

114 personnes (totalité du panel) ont répondu au questionnaire. Parmi eux, la population A désignera les individus pratiquant l'activité 3 (utilisation de LinkedIn comme outil de travail) et la population $\mathrm{B}$ désignera les individus pratiquant l'activité 4 (ajout d'un descriptif relatif à l'employeur sur le profil). La population A-B correspond donc à l'échantillonnage qui nous intéresse ici. Composée de 57 individus, la population A-B se structure en trois tiers (figures 3) : la population $\mathrm{A}^{2}$ (elle correspond aux individus n'appartenant qu'à la population $\mathrm{A}$, de manière exclusive), la population $\mathrm{B}^{2}$ (elle correspond aux individus n'appartenant qu'à la population $\mathrm{B}$, de manière exclusive), et la population $\mathrm{AB}^{2}$ (elle correspond aux individus appartenant à la fois au groupe $\mathrm{A}$ et au groupe $\mathrm{B}$ ).

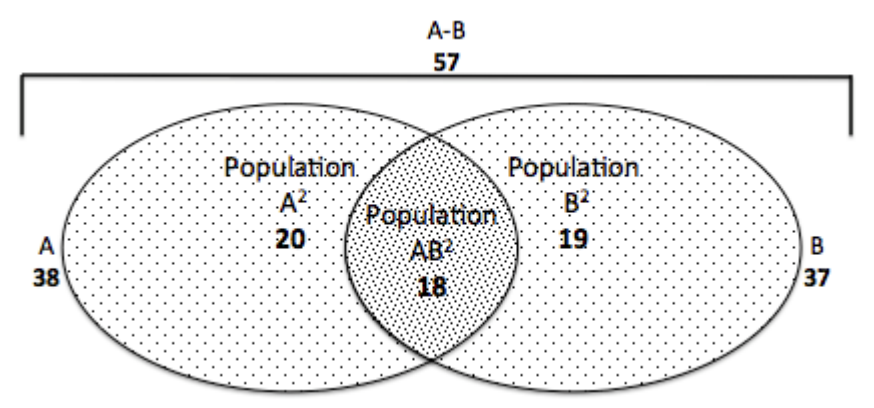

Figure 3. Nombre d'individus pour chaque groupe de population

Les professionnels RH représentaient $31 \%$ de la totalité du panel. Or, ils sont surreprésentés cdez les A-B : ils composent $50 \%$ du groupe A et $57 \%$ du groupe B. Cette représentativité est encore marquée dans le groupe $\mathrm{AB}^{2}$ où ils sont présents à hauteur de $77 \%$. Par ailleurs, les chiffres indiquent que la population $\mathrm{AB}^{2}$ est composée à $78 \%$ de consultants $\mathrm{RH}$.

\subsubsection{Implications stratégiques : efficacité et identité individuelle professionnelle}

Toute la population A a affirmé (sans exception, et sans aucun écart de réponse) que l'utilisation de LinkedIn comme outil de travail se faisait notamment pour gagner en efficacité au travail (tableau 3). L'aspect stratégique à caractère collectif de l'activité 3 a donc bien été confirmé. Pour la population $\mathrm{A}$, il semblerait par ailleurs que concilier une stratégie collective avec une stratégie individuelle soit largement possible, puisque $79 \%$ de ces répondants affirment que l'activité 3 (l'utilisation de LinkedIn comme outil de travail) leur permet également d'asseoir leur identité professionnelle. En revanche, pour la population $\mathrm{B}$ la stratégie collective est assez en retrait comparativement à la stratégie individuelle (asseoir son identité professionnelle) avec un écart de $24 \%$. Ceci peut nous laisser présager que l'ajout d'un descriptif sur le profil marque une tension entre stratégie collective et individuelle. 


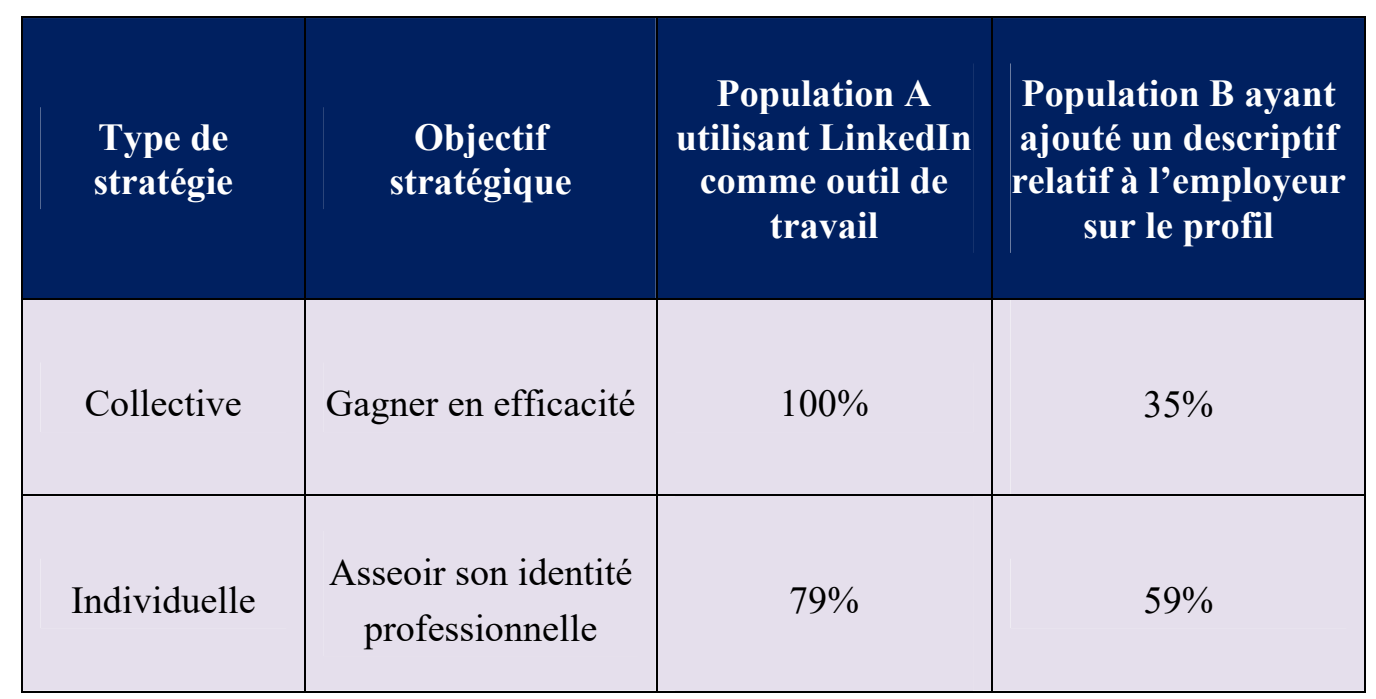

Tableau 3. Résultats du questionnaire préalable

\subsection{Résultats des entretiens}

\subsubsection{Entretiens auprès des professionnels $R H$}

Sur la base des résultats du questionnaire, les entretiens de recherche ont été réalisés auprès de professionnels RH. Une large place a été laissée aux consultants RH particulièrement concernés par les activités réalisées en interne. 4 recruteurs et 4 consultants $\mathrm{RH}$, tous Québécois ${ }^{9}$, ont ainsi été interviewés lors d'entretiens directifs (tableau 4) menés entre mars et juin 2016.

\begin{tabular}{|c|c|c|c|c|}
\hline Interviewé ${ }^{10}$ & Fonction & $\begin{array}{l}\text { Niveau de } \\
\text { diplôme }\end{array}$ & Âge & Sexe \\
\hline Gilles & Recruteur & $\mathrm{Bac}+3$ & 37 & M \\
\hline Louise & Recruteur & $\mathrm{Bac}+4$ & 42 & $\mathrm{~F}$ \\
\hline Henriette & Recruteur & $\mathrm{Bac}+3$ & 28 & $F$ \\
\hline Fabien & Recruteur & $\mathrm{Bac}+3$ & 30 & $\mathrm{M}$ \\
\hline Clara & Consultant RH & $\mathrm{Bac}+2$ & 50 & $\mathrm{~F}$ \\
\hline Laurence & Consultant RH & $\mathrm{Bac}+3$ & 59 & $\mathrm{~F}$ \\
\hline Denis & Consultant RH & $\mathrm{Bac}+3$ & 34 & $\mathrm{M}$ \\
\hline Robert & Consultant RH & $\mathrm{Bac}+3$ & 56 & M \\
\hline
\end{tabular}

Tableau 4. Caractéristiques des interviewés

Les données des entretiens ont d'abord été traitées de manière quantitative. Un nuage de mots (figure 5) met en évidence que l'entreprise et l'employeur occupent une place prépondérante dans les discours : ils prévalent sur le mot «profil». On peut en déduire que la stratégique collective (utiliser LinkedIn pour le bénéfice de son employeur) est dominante. En arrière plan surgissent les thèmes de la quête d'opportunités et la question de l'emploi. Un conflit de priorité semble donc se jouer entre la

9 Ces entretiens de recherche ont pris place dans le cadre plus large d'une comparaison des pratiques des utilisateurs Français et Québécois. Sur la question de la conciliation entre stratégie collective et individuelle, les entretiens menés avec les Français n'ont eu qu'un caractère exploratoire. La question portant sur un éventuel conflit entre ces deux aspects n'a donc été formulée explicitement que lors de la deuxième phase de mon enquête, réalisée auprès de Canadiens. Seule cette partie-là de l'enquête était donc exploitable ici. C'est la raison pour laquelle les interviewés sont tous ici nationalité Québécoise. 
stratégie individuelle et collective. Cette analyse est confirmée par l'analyse thématique des entretiens, même si elle reste à nuancer.

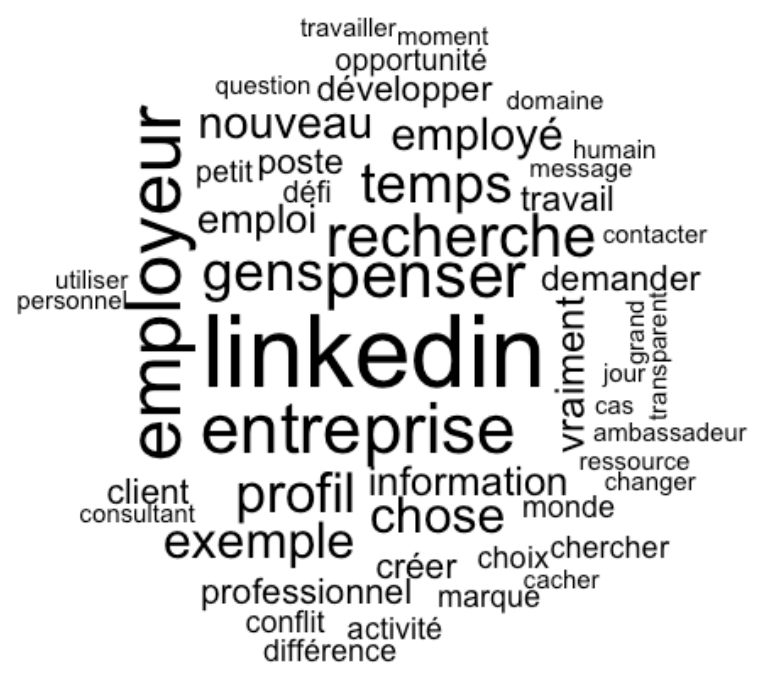

Figure 5. Nuage de mots réalisé par IRaMuTeq à partir du discours des consultants $R H$ et des recruteurs

En effet, 7 interviewés sur 8 ont précisé les raisons pour lesquelles il pouvait y avoir une tension entre stratégie collective et individuelle (figure 4). Le fait d'indiquer explicitement être à la recherche d'un emploi comptent parmi les motifs de tension les plus mentionnés. Ne pas préciser sur son profil que l'on est ouvert aux opportunités professionnelles s'impose alors comme une omission diplomatique ou comme une manière de faire preuve de tact, deux techniques permettent de garder la face et d'éviter les représentations contradictoires (Goffman, 1974, p. 9). Il arrive aussi que le risque d'entrer en compétition directe avec l'employeur se présente. Plus ponctuellement, la nécessité de faire un choix quant à sa mise en scène sur un réseau à l'adressage large a été évoquée : " c'est vraiment général, tu ne peux pas sélectionner en disant : je veux qu'il y ait juste $90 \%$ de mon réseau mais pas les 10 autres » (Louise). Enfin, les conflits de valeur ou d'intérêt entre l'employeur et l'employeur peuvent marquer pour Clara un clivage entre deux pôles stratégiques.

La question de la mise en valeur de soi dans une perspective stratégique individuelle de recherche d'emploi a été la plus abordée. Henriette souligne la différence entre se mettre en scène pour son employeur actuel ou pour rechercher un emploi : «je vais me faire quelque-chose de beau mais quelque-chose pour moi maintenant, pour montrer que là ce n'est plus la recruteuse mais la candidate »

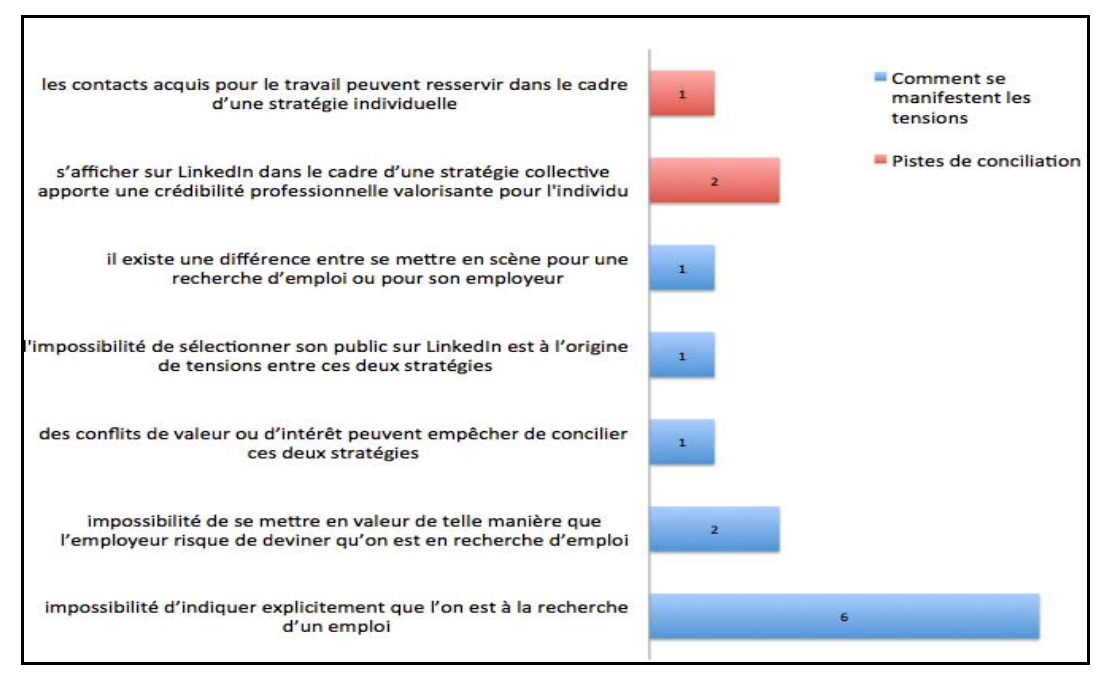

Figure 4. Existe-t-il des tensions entre l'utilisation de Linkedln par rapport à votre employeur et l'utilisation de Linkedln pour vous-même? 
Fabien préfère quant à lui éviter de mettre en valeur ses succès au risque de laisser croire à son employeur qu'il recherche un emploi ailleurs : "J'ai gagné plusieurs prix avec la compagnie, mais les gens qui vont mettre ça c'est qu'ils sont en recherche d'emploi [...] donc je me demande si je le mets là, si la compagnie va penser que je suis en train de valoriser mon profil pour... ». Robert abonde également dans ce sens au travers d'une remarque d'ordre général : "s'il ne veut pas que l'employeur soit au courant de peur de se faire licencier, il va être limité, il ne pourra pas mettre l'information, donner toutes ses compétences et dire: je suis disponible pour aller travailler ». Ceci peut-être interprété comme une certaine forme d'«absence de reconnaissance par les entreprises de l'identité pour soi » (Doray, 1992, p. 310) mais également comme une impossibilité pour l'utilisateur d'actualiser son rôle au risque de ne pas respecter un "système de places » (Goffman, 1974). La négociation de la face prévaut alors sur les aspirations plus personnelles de l'utilisateur. Au total, 6 interviewés ont fait part de l'impossibilité d'indiquer sur le profil le fait de rechercher un nouvel emploi : « je ne marque pas en recherche de nouveaux défis non plus, je ne crois pas que c'est quelque chose qui doit être marqué », confie Denis.

On retiendra donc que le fait d'être impliqué dans une stratégie collective inhibe ou peut potentiellement inhiber l'expression de quatre référents identitaires (tableau 5) : les valeurs, les compétences, les distinctions honorifiques et les motivations.

\begin{tabular}{|c|c|c|c|}
\hline $\begin{array}{c}\text { Catégorie de } \\
\text { référents }\end{array}$ & $\begin{array}{c}\text { Sous- } \\
\text { catégorie de } \\
\text { référents }\end{array}$ & Référents & Source \\
\hline Culturels & $\begin{array}{c}\text { Système } \\
\text { culturel }\end{array}$ & $\begin{array}{c}\text { Valeurs de } \\
\text { l'individu }\end{array}$ & Clara \\
\hline Psychosociaux & $\begin{array}{c}\text { Attributs de } \\
\text { valeur sociale }\end{array}$ & Compétences & Robert \\
\hline Psychosociaux & $\begin{array}{c}\text { Attributs de } \\
\text { valeur sociale }\end{array}$ & $\begin{array}{c}\text { Prix obtenu } \\
\text { au sein de la } \\
\text { compagnie }\end{array}$ & Fabien \\
\hline Psychosociaux & $\begin{array}{c}\text { Potentialités } \\
\text { de devenir }\end{array}$ & $\begin{array}{c}\text { Motivation } \\
\text { professionnel } \\
\text { le (recherche } \\
\text { d'un emploi) }\end{array}$ & $\begin{array}{c}\text { Denis / Gilles / } \\
\text { Louise / } \\
\text { Fenriette / } \\
\text { Fabien / Robert }\end{array}$ \\
\hline
\end{tabular}

Tableau 5. Référents identitaires dont l'expression peut être inhibée par la stratégie collective

Malgré toutes ces tensions, des pistes de conciliation entre stratégie individuelle et collective ont été formulées par 2 interviewés. Ici, le thème du renforcement identitaire est apparu en filigrane. Pour Clara, Laurence et Gilles l'utilisation de LinkedIn dans le cadre d'une stratégie collective peut apporter une crédibilité dont l'individu peut tirer partie individuellement en externe : «c'est une image qu'ils vont avoir de moi de quelqu'un de professionnel» (Gilles) ; «personnellement ça m'a grandement servi, ça m'a permis de me faire connaitre et de me faire voir comme quelqu'un de reconnu dans le domaine des ressources humaines dans ma région et même un petit peu au-delà » (Clara).

Mais surtout, on observe que toutes les sources de tensions sont relatives au contenu même du profil, du côté du déclaratif (figure 4), tandis que l'une des deux pistes de conciliations renvoie à l'utilisation de LinkedIn pour accumuler des contacts (du côté de l'agir). On peut en déduire que la faculté à pouvoir concilier une stratégie collective avec une stratégie individuelle dépend en grande partie du type d'activité pratiquée : il semblerait que la conciliation soit d'avantage possible lorsque l'activité relève de l'action (prospecter, agrandir son réseau) que lorsqu'elle relève de l'affichage. Gilles témoigne en ce sens : "quelqu'un avait remarqué qu'il était toujours à la recherche de (C) 2017 ISTE OpenScience - Published by ISTE Ltd. London, UK - openscience.fr 
nouveaux défis sur son LinkedIn, et puis il [l'employeur] lui a fait un commentaire à cet effet-là. Ils ne lui ont pas demandé de l'enlever mais ils lui ont dit qu'ils l'avaient remarqué et que ce n'était pas apprécié. [...] Je pense qu'il faut plus envoyer des demandes ciblées quand on est en recherche et puis envoyer des mails plutôt que d'indiquer sur son profil que l'on est en recherche d'emploi ».

Ce point est illustré par l'analyse des similitudes des discours (ADS) à l'aide le logiciel IRaMuTeQ (figure 5). La branche "profil» (du côté de l'affichage) est clairement distincte de la branche « recherche» (du côté de l'agir). De plus, le profil comme lieu d'affichage pour rester ouvert aux opportunités (branche de gauche) dans une perspective stratégique individuelle se distingue du thème de l'employeur (branche du bas). Enfin, la branche de droite révèle que LinkedIn est utilisé comme un instrument de "recherche» dans une perspective à la fois collective (chercher de "nouveaux» « clients ») et individuelle (chercher de « nouveaux » « défis », c'est-à-dire un emploi).

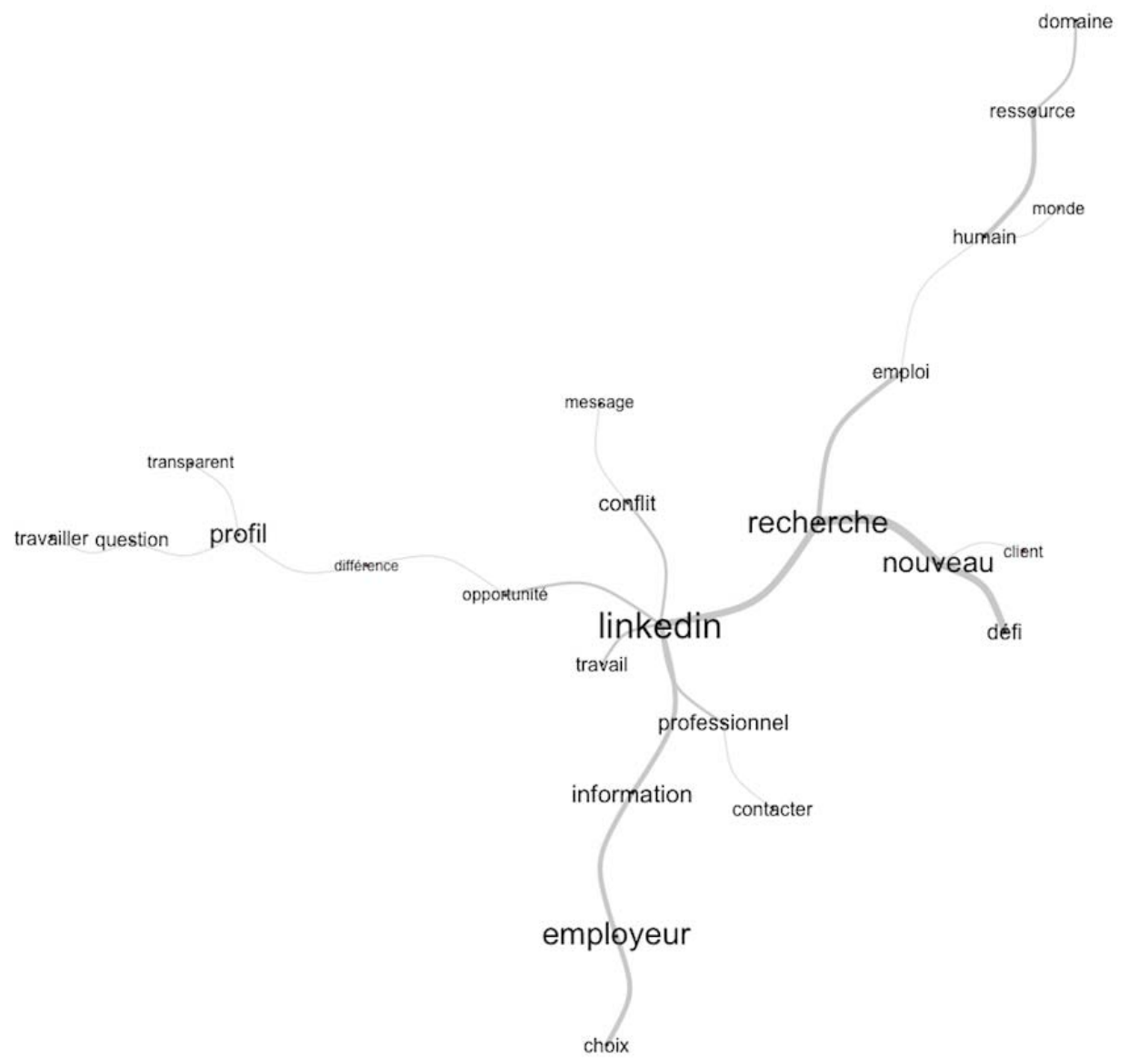

Figure 5. Analyse Des similitudes (ADS) des discours des consultants $R H$ et des consultants par IRaMuTeq

\subsubsection{Entretiens auprès des experts Linkedln}

Les résultats des entretiens menés avec les experts ont confirmé que la clef de conciliation entre stratégie collective et individuelle se situe, non pas seulement dans une capacité à ménager se mise en scène sur le profil, mais dans une habile dissociation entre le dire et l'agir permettant d'entretenir deux façades identitaires, l'une orientée vers le collectif l'autre vers l'individuel. Un expert LinkedIn nous raconte :

" ça demande beaucoup d'astuce mais c'est tout à fait faisable, je le fais en ce moment pour deux clients qui font en même temps recherche d'emploi marché caché et pourtant quand on voit leur profil ils sont à fond dans le promotion de leur entreprise, mais on ne le voit pas du tout, on ne le voit absolument pas de l'extérieur, mais de l'intérieur du profil LinkedIn on va chercher vraiment les 
opportunités d'emploi. [..] vous ne le voyez pas vous : vous allez sur le profil, vous ne verrez pas qu'il cherche un autre job. Vous verrez que c'est une super promotion de son entreprise. Mais de l'intérieur, en caché, on chasse des postes pour partir de l'entreprise. On peut tout à fait atteindre les deux objectifs en même temps. » (expert 1)

C'est donc au travers de l'action que le profil LinkedIn semble pouvoir regagner tout son aspect stratégique individuel, même lorsque l'individu s'affiche explicitement au nom d'un collectif. En somme, l'orientation de l'activité, soit vers l'agir soit vers la représentation, permet de changer de paradigme, à la fois au niveau identitaire et stratégique. L' " astuce », c'est-à-dire le niveau de maîtrise de LinkedIn, conditionne on le comprend la mise en œuvre de cette technique décrite par l'expert 1 . Se former à l'utilisation du réseau s'impose alors comme une condition nécessaire pour se dégager du primat de la stratégie collective.

En ce qui concerne le deuxième expert, il rapporte la capacité à concilier une stratégie individuelle et collective au pays d'appartenance :

"l'employeur voit un avantage pour que l'employé développe sa marque personnelle et il voit que l'employé est ambassadeur pendant le temps qu'il est employé chez lui. Mais ça je pense qu'en France c'est la même chose, en tout cas en Suisse c'est encore très difficile à faire comprendre aux entreprises : ils voient toujours le danger au lieu de voir le bénéfice » (expert 2).

Cette remarque concernant l'ancrage culturel peut constituer une hypothèse de recherche intéressante pour une prochaine enquête et dénote le clivage qui existe actuellement, en France et en Suisse, entre la stratégie individuelle et la stratégie collective.

\section{Synthèse et mise en perspective}

Notre enquête a débouché sur trois constatations importantes. Premièrement, l'implication de l'utilisateur dans une stratégie collective inhibe l'expression de certains référents identitaires, dont la plupart se situent dans la catégorie des référents psychosociaux. L'utilisateur est en effet avant tout soucieux de conserver des relations diplomatiques avec son employeur, de faire preuve de tact et de respecter un système de places. Ce faisant, la négociation de la face prévaut sur les aspirations personnelles de l'utilisateur. Deuxièmement, la faculté à pouvoir concilier une stratégie collective avec une stratégie individuelle dépend en grande partie du type d'activité pratiquée : il semblerait que la conciliation soit d'avantage possible lorsque l'activité relève de l'action (prospecter, agrandir son réseau) que lorsqu'elle relève de l'affichage. Troisièmement, pour poursuivre une double stratégie, à la fois individuelle et collective, il peut être nécessaire de scinder le déclaratif (le contenu du profil) et l'agir (le réseautage, l'envoi de messages) en orientant le premier vers une stratégie collective et l'autre vers une stratégie individuelle. Elargir son réseau, envoyer des inmails ${ }^{11}$ pour renforcer des relations se fait alors «en sous-marin », loin du regard du collectif. Cette invisibilité permet à l'individu de déployer sa propre stratégie sans se mettre en porte à faux par rapport au collectif.

Ici, on remarquera donc que le travail du réseau « en sous-marin » se situe à rebours du concept de «public display of connections»: boyd et Donath désignent ainsi la mise en visibilité publique des connexions comme une «vérification implicite de l'identité » (Donath \& Boyd, 2004, p. 73). Cette mise en visibilité « peut être vue comme le signal de la fiabilité de la revendication identitaire » (ibid.) et s'impose comme une méthode permettant d'affirmer son identité. Dans un phénomène inverse, tisser des relations "en sous-marin », c'est-à-dire des relations invisibles, peut permettre à l'individu impliqué dans un collectif d'affirmer librement son identité sans être tenu à des restrictions générées par la visibilité.

11 L'InMail permet de contacter un utilisateur par le biais de la plate-forme Linkedln. II n'est pas nécessaire pour ce faire de connaître son adresse mail classique. 
Par ailleurs, la distinction que j'établis entre les activités de l'ordre du déclaratif et les activités de l'ordre de l'agir évoque l' opposition entre l'identité déclarative et l'identité agissante (Georges, 2009). Cependant, pour que ce parallèle soit exact, il me semble nécessaire de revisiter la notion d'identité agissante décrite comme le "relevé explicite des activités de l'utilisateur par le Système» (ibid. p. 168). Je lui préfère ici celle « d'identité en actes ». L' « identité en actes », telle que je la définis, serait alors constituée des différentes activités des utilisateurs ne donnant lieu à aucune trace explicite sur le système et permettant, grâce aux interactions interpersonnelles qu'elles génèrent, de mettre en avant différents référents identitaires, tels que la motivation, des valeurs ou des compétences.

Enfin, pour compléter cette approche par référents de l'identité professionnelle personnelle sur LinkedIn il pourrait être intéressant d'adopter une perspective Ricoeurienne de construction de soi par l'identité narrative subjective (Ricoeur, 1990). La déclinaison de différentes expériences sur le profil s'imposerait alors comme un mode narratif synthétique de l'histoire professionnelle d'un unique locuteur, la subjectivité résidant alors dans le choix que peut faire l'utilisateur de décrire son expérience professionnelle en adoptant une perspective particulière, notamment sectorielle, culturelle ou en termes de catégorie de compétences. Sur cette base, il serait intéressant de rechercher sur les profils LinkedIn des indices de subjectivité, puis de comprendre dans quelles mesures ces indices sont au service d'une stratégie collective ou individuelle. On pourrait alors comprendre dans quelles mesures l'individu impliqué dans une stratégie collective peut s'approprier son histoire, étape incontournable à la «construction de soi comme professionnel » et au " positionnement identitaire » (Beckers, 2007, p. 301-302).

\section{Bibliographie}

Agostinelli, S., Arvanitakis, S., \& Ouvrard, M. (2015). E-réputation et profil de compétences relationnelles. In OMSRP (Ed.), Médias sociaux et relations publiques (pp. 9-21). Québec (Québec): Presses de l'Université du Québec.

Audrerie, J.-B. (2015, May 25). Le recrutement inbound en 5 notions essentielles. Retrieved May 17, 2017, from $\mathrm{http} / / /$ futurstalents.com/marketing-rh/marketing-digital-rh/le-recrutement-inbound-en-5-notions-essentielles/

Backhaus, K. B. (2004). An Exploration of Corporate Recruitment Descriptions on Monster.com. Journal of Business Communication, 41(2), 115-136.

Beckers, J. (2007). Compétences et identité professionnelles : l'enseignement et autres métiers de l'interaction humaine. Bruxelles: De Boeck.

Boudokhane-Lima, F., \& Felio, C. (2015). Les usages professionnels des TIC: des régulations à construire. Communication et organisation, (48), 139-150.

Bughin, J., \& Chui, M. (2010, December). The rise of the networked enterprise: Web 2.0 finds its payday. The McKinsey Quarterly.

Cardon, D. (2008). Le design de la visibilité: Un essai de cartographie du web 2.0. Réseaux, 152(6), 93.

Cardon, D. (2013). L’identité comme stratégie relationnelle. Hermès, La Revue, (53), 61-66.

Chartier, M. (2013). Guide complet des réseaux sociaux. Paris: First Interactive.

Coutant, A., \& Stenger, T. (2010). Processus identitaire et ordre de l'interaction sur les réseaux socionumériques. Les Enjeux de L'information et de La Communication, 2010(1), 45-64.

Crussière, L. (2015, November 4). 15 défauts qui rendront votre profil Linkedin efficace. Retrieved from https://blog.agencenile.com/15-défauts-à-avoir-pour-un-profil-linkedin-efficace

Donath, J., \& Boyd, D. (2004). Public displays of connection. Bt Technology Journal, 22(4), 71-82.

Doray, P. (1992). Claude Dubar, La socialisation, construction des identités sociales et professionnelles, Paris, Armand Colin, 1991, 278 p. Cahiers de recherche sociologique, (18-19), 308.

Dubar, C. (1992). Formes identitaires et socialisation professionnelle. Revue française de sociologie, 33(4), 505-529.

Felio, C. (2013). Visibilité numérique des cadres d'entreprise. Communication et organisation, (44), 123-132. 
Felio, C. (2016). Par-delà les pratiques individuelles: l'idéologie managériale comme armature de l'autodiscipline des cadres équipés de TIC. Travailler, (35), 191-212.

Fondeur, Y. (2006). Recrutement par Internet : le dilemme transparence-bruit. Personnel, (472), 46-48.

Georges, F. (2009). Représentation de soi et identité numérique. Réseaux, 154(2), 165-193.

Girard, A. (2012). L'intégration des médias sociaux dans les stratégies d'e-GRH: le cas du recrutement. Université Montpellier 2.

Girard, A., Fallery, B., \& Rodhain, F. (2011). L'apparition des médias sociaux dans l'e-GRH: gestion de la marque employeur et e-recrutement. In Actes du congrès AIM (pp. 1-18).

Goffman, E. (1974). Les Rites d'interaction. Paris: Minuit.

Goffman, E. (1975). Stigmate : Les usages sociaux des handicaps (Editions de Minuit). Paris: Les Editions de Minuit.

Kriegk, J.-S. (2012, September 5). Les réseaux sociaux, levier de la performance des entreprises françaises. Retrieved June 12, 2017, from http://archives.lesechos.fr/archives/cercle/2012/09/05/cercle_53508.htm

Larroche, V. (2014). Quelle visibilité professionnelle pour un salarié ambassadeur 2.0 auprès des recruteurs potentiels?. Communication \& Organisation, (44), 53-64.

Larroche, V. (2015). Les médiateurs de confiance comme gage de crédibilité des candidats - Le cas des réseaux sociaux professionnels numériques. In C. Alcantara, E-réputation: regards croisés sur une notion émergente. Issy-lesMoulineaux: Gualino Editions.

Lievens, F., van Dam, K., \& Anderson, N. (2002). Recent trends and challenges in personnel selection. Personnel Review, 31(5), 580-601.

MacAfee, A. (2006, May 27). Enterprise 2.0, version 2.0. Retrieved June 15, 2017, from http://andrewmcafee.org/2006/05/enterprise_20_version_20/

Malita, L., Badescu, I., \& Dabu, R. (2010). Culture tips of online job searching. Procedia - Social and Behavioral Sciences, 2(2), 3070-3074.

Mucchielli, A. (2013). L’identité (Que sais-je ?). Presses Universitaires de France.

RégionsJob. (2013). Marque employeur et recrutement 2.0, exemples et bonnes pratiques, 2ème édition. RégionsJob.

Ricoeur, P. (1990). Soi-même comme un autre. Paris: Seuil.

Roberts, S. J., \& Roach, T. (2008). Social Networking Web Sites and Human Resource Personnel: Suggestions for Job Searches. Business Communication Quarterly, 72(1), 110-114.

Roll, R. (2010). Développement durable : exporter au prix gagnant grâce au « sourcing en réseaux ». Géoéconomie, 52(1), 33.

Stenger, T., \& Coutant, A. (2010). Les réseaux sociaux numériques: des discours de promotion à la définition d'un objet et d'une méthodologie de recherche. Hermes-Journal of Language and Communication Studies, 44, 209-228.

Taboada-Leonetti, I. (1998). Stratégies identitaires et minorités : le point de vue du sociologue. In Stratégies identitaires (pp. 43-83). Presses Universitaires de France. 